\title{
EQUIVALENCE AND SLICE THEORY FOR SYMPLECTIC FORMS ON CLOSED MANIFOLDS
}

\author{
R. C. SWANSON AND C. C. CHICONE
}

\begin{abstract}
In this paper, a study is made of the pullback action of the diffeomorphism group on the totality of symplectic forms on a compact manifold. For this action, the orbit is shown to be a smooth (Banach) manifold consisting of a denumerable union of submanifolds, each lying in a fixed cohomology class.

In addition, a precise characterization is given of those symplectic manifolds for which there is a local factorization of the pullback action in the sense of a transverse "slice" of closed 2-forms, invariant under the group of symplectic diffeomorphisms.
\end{abstract}

0. Introduction. A symplectic structure or form is a nondegenerate closed 2 -form $\omega$ on a compact connected smooth manifold $M$. Two symplectic forms $\omega$ and $\omega^{\prime}$ are equivalent if $\omega^{\prime}=f^{*} \omega$ for some diffeomorphism $f: M \rightarrow M$. J. Moser [6] was the first to make substantial progress on the problem of deciding when two symplectic structures are equivalent. His landmark discovery was that two symplectic structures which are homotopic and cohomologous are equivalent. In this article, while relying to a certain extent on his approach, we attack the problem from an infinite dimensional point of view. Thus, we study a Banach or Fréchet "Lie group" (the group of Sobolev or smooth diffeomorphisms of $M$ ) acting on a smooth manifold (the closed 2-forms). This program was exploited by Ebin [1] to construct a slice for the pullback action of the diffeomorphism group on the manifold of metrics. The deepest (and unsolved) problem is to characterize the group orbits for the action of pullback on closed forms. In addition to Moser's result, it is known that differential forms on a compact manifold are never stable under pullback (Golubitsky and Tischler [3]). Given this constraint, one would like to know whether a slice (a local factorization of the action) can be constructed for the action of the diffeomorphism group.

We characterize the symplectic manifolds which admit a slice (Theorem 2.3). The lack of a slice in most cases (e.g. the $2 n$-torus) contrasts vividly with Ebin's result in the metric case. In addition, we demonstrate that the orbit (equivalence class) of a symplectic form is itself a smooth manifold composed of a denumerable union of submanifolds each lying in a fixed cohomology class (Theorem 3.7).

Received by the editors March 31, 1978.

AMS (MOS) subject classifications (1970). Primary 58A10; Secondary 58D05.

Key words and phrases. Symplectic forms, pullback, slice. 
1. Notation and terminology. $M$ is a fixed compact connected smooth manifold with symplectic structure $\omega$. $\bigodot^{\infty}\left(\Lambda^{p}\right)$ denotes the differential $p$-forms on $M, Z^{p}$ the closed subspace of closed $p$-forms and $H^{p}(M)$ the $p$ th de Rham cohomology group. If $\mathscr{D}$ is the group of smooth orientation preserving diffeomorphisms of $M$ define for $\theta$ in $Z^{p}$ the orbit map $\alpha_{\theta}$ : $\mathscr{D} \rightarrow Z^{p}$ by $f \rightarrow f^{*} \boldsymbol{\theta}$.

To avoid the technical difficulties associated with the analysis on Fréchet manifolds, we enlarge the smooth objects defined above to the appropriate Sobolev (Hilbert) manifolds. In place of $\bigodot^{\infty}\left(\Lambda^{p}\right)$ one has $\mathcal{H}^{s}\left(\Lambda^{p}\right), Z_{s}^{p}$ extends $Z^{p}$, and $\mathscr{D}$ extends to $\mathscr{Q}^{s}$; the topological group of $\mathcal{H}^{s}$ diffeomorphisms of $M$. Unless otherwise indicated we require $s>n / 2+1$ to assure that elements of $\mathcal{H}^{s}\left(\Lambda^{p}\right)$ and $\mathscr{Q}^{s}$ are $\mathcal{C}^{1}$ and that $\mathscr{Q}^{s}$ is a smooth Hilbert manifold. For more details see Ebin [1] or Ebin and Marsden [2].

From the Hodge-de Rham decomposition theorem $Z^{p}$ is isomorphic to the direct sum $d\left[\mathcal{H}^{s+1}\left(\Lambda^{p-1}\right)\right] \oplus H^{p}$ where $d$ is the exterior derivative and $H^{p}$ is the space of harmonic $p$-forms with respect to some Riemannian metric on $M$. Thus $H^{p}$ is isomorphic to the $p$ th de Rham group $H^{p}(M)$.

For $X$ in $\chi(M)$, the smooth vector fields on $M$, (or $\chi^{s}(M)$ the $\mathcal{F}^{s}$ vector fields on $M$ ) let $f_{t}$ be a curve of diffeomorphisms of $M$ tangent to $X$ and define the Lie derivative of a $p$-form $\theta$ in the direction $X$ by

$$
L_{X} \theta=\frac{d}{d t}\left(f_{t}^{*} \theta\right)_{t=0}
$$

Equivalently, we have Cartan's formula

$$
\left.\left.L_{X} \theta=d(X\lrcorner \theta\right)+X\right\lrcorner d \theta
$$

where $\lrcorner$ denotes the interior product.

2. The local theory. Fix a smooth $p$-form $\theta$. The orbit map $\alpha_{\theta}$ extends uniquely to a smooth mapping $\alpha_{\theta}^{s}$ : $\mathscr{Q}^{s+1} \rightarrow \mathcal{H}^{s}\left(\Lambda^{p}\right)$ [2]. The differential $T_{\text {id }} \alpha_{\theta}^{s}: \chi^{s+1} \rightarrow \mathcal{H C}\left(\Lambda^{p}\right)$ is given by $T_{\text {id }} \alpha_{\theta}^{s}(X)=L_{X} \theta$. For $\omega$ a closed 2-form Cartan's formula gives $\left.L_{X} \omega=d(X\lrcorner \omega\right)$. Hence, the range of $T_{\text {id }} \alpha_{\theta}^{s}$ consists of all exact 2 -forms if $\omega$ is symplectic.

Let $\mathscr{D}_{0}^{s+1}$ denote the open subgroup of $\mathscr{O}^{s+1}$ given by the identity component which, in fact, corresponds to the set of diffeomorphisms isotopic to the identity. For us, the restriction of $\theta_{\omega}^{s}$ to $\mathscr{Q}_{0}^{s+1}$ constitutes the local theory, i.e. local in $\mathscr{Q}^{s+1}$. Since $\alpha_{\omega}^{s}$ is smooth, the orbit set $\theta_{0}^{s}=\left\{f^{*} \omega \mid f \in\right.$ $\left.\mathscr{Q}_{0}^{s+1}\right\}$ is a connected subset of the orbit $\theta_{\omega}^{s}=\left\{f^{*} \omega \mid f \in \mathscr{Q}^{s+1}\right\}$. Amalgamating the results of Moser [6] and Ebin and Marsden [2, Lemma 4.1] we now show that a symplectic form is stable in its cohomology class; a notion first formulated by Martinet [4].

THEOREM 2.1. Fix $s>\frac{1}{2} \operatorname{dim} M$. If $\omega$ is a smooth symplectic form on $M$ then the orbit $\theta_{0}^{s}$ is a smooth connected submanifold of the space of closed forms $Z_{s}^{2}$ with codimension $k=b_{2}$, the second Betti number. In fact, $\theta_{0}^{s}$ is open in the 
cohomology class $[\omega]_{s}=\left\{\omega+d \alpha \mid \alpha \in \mathcal{H}^{s+1}\left(\Lambda^{1}(M)\right)\right\}$. The same conclusions hold in the category of smooth forms.

Proof. Since $\omega$ is symplectic the differential of the orbit map $\alpha_{\omega}^{s}$ at the identity maps surjectively onto the exact 2 -forms. As the pullbacks of $\omega$ by homotopic maps are cohomologous the image $\alpha_{\omega}^{s}\left(\mathscr{Q}_{0}^{s+1}\right)$ lies in $[\omega]_{s}$. Therefore, $\alpha_{\omega}^{s}: \mathscr{Q}_{0}^{s+1} \rightarrow[\omega]_{s}$ is a submersion onto its image and the result follows from the implicit function theorem. For the smooth case, note that $\mathscr{D}_{0}=$ $\bigcap_{s>n / 2} \mathscr{Q}_{0}^{s+1}$ and give $\theta_{0}=\bigcap_{s>n / 2} \theta_{0}^{s}$ the limit topology. Then, the result follows from the naturality of the construction. For details see Omori [7]. Q.E.D.

Next we explicitly exhibit the orbit $\theta_{0}^{s}$ as a smooth homogeneous space of $\mathscr{Q}_{0}^{s+1}$. For this, let $\delta p_{0}^{s}$ denote the subgroup $\delta p_{0}^{s}=\left\{f \in \mathscr{D}_{0}^{s+1} \mid f^{*} \omega=\omega\right\}$. $\delta p_{0}^{s}$ is a closed subgroup of $\mathscr{Q}_{0}^{s+1}$, hence $\mathfrak{Q}_{0}^{s+1} / \delta p_{0}^{s+1}$ inherits the identification topology from the canonical projection.

THEOREM 2.2. The quotient $\mathfrak{D}_{0}^{s+1} / \delta p_{0}^{s+1}$ admits a differential structure such that the projection $\Pi_{s}: \mathscr{Q}_{0}^{s+1} \rightarrow \mathscr{Q}_{0}^{s+1} / \delta p_{0}^{s+1}$ is a submersion of Hilbert manifolds and the canonical coset map $A: \mathscr{Q}_{0}^{s+1} / \delta p_{0}^{s+1} \rightarrow \Theta_{0}^{s}$ given by $\delta p_{0}^{s+1} \cdot f \rightarrow$

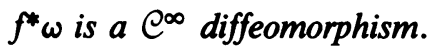

Proof. Since $\mathscr{Q}_{0}^{s+1}$ is a topological group acting transitively on $\theta_{0}^{s}$, the map $A$ is continuous and bijective (Montgomery and Zippin [5]). Since $\alpha_{\omega}^{s}=$ $A \circ \Pi_{s}$ and $\alpha_{\omega}^{s}$ is an open mapping it follows that $A$ is open and hence a homeomorphism. Give $\mathscr{Q}_{0}^{s+1} / \delta p_{0}^{s+1}$ the differential structure that makes $A$ a diffeomorphism and note that then $\Pi_{s}=A^{-1} \circ \alpha_{\omega}^{s}$ is a submersion onto the quotient space. Q.E.D.

Remark. $\Pi_{s}$ admits a smooth local cross section for $s>n / 2$. Also, $\Pi=\lim \Pi_{s}$ admits a local continuous cross section in the smooth case.

For a group action $G \times N \rightarrow N$ let $x \in N$ and let $G_{x}$ denote the stabilizer subgroup at $x$. A slice at $x$ is a subset $S_{x}$ of $N$ containing $x$ together with a local cross section $\eta: G / G_{x} \rightarrow G$ defined in a neighborhood $U$ of $G_{x}$ such that:

(i) $G_{x} S_{x}=\left\{g \cdot s \mid g \in G_{x}, s \in S_{x}\right\}=S_{x}$.

(ii) If $g S_{x} \cap S_{x} \neq \varnothing$, then $g \in G_{x}$.

(iii) The map $(u, s) \rightarrow \eta(u) \cdot s$ defines a local homeomorphism of $U \times S_{x}$ onto a neighborhood of $x$ in $N$.

The pullback action of $\mathscr{D}$ on the manifold of Riemannian metrics admits a slice through each metric. In the next theorem we classify the symplectic manifolds which admit a slice.

THEOREM 2.3. The symplectic manifold $M$ admits a slice $\Sigma_{\omega}$ at the symplectic form $\omega$ if and only if $H^{2}(M)=R$.

Proof. Assume $\Sigma_{\omega}$ is a slice at $\omega$ and choose $\omega^{\prime} \in \Sigma_{\omega}$. Let $\delta p_{0}^{\prime}=\delta p_{\omega^{\prime}} \cap$ $\mathscr{D}_{0}$ and choose $f \in \delta p_{0}^{\prime}$. Then, $f^{*} \omega^{\prime}=\omega^{\prime}$ and by (ii) $f \in \delta p_{0}$, i.e. $\delta p_{0}^{\prime} \subseteq \delta p_{0}$. 
Choose a Darboux chart for $\omega^{\prime}$, i.e. a coordinate neighborhood $V \subset R^{2 k}$ in which $\omega^{\prime}$ is constant. Let the local representative of $\omega^{\prime}$ (resp. $\omega$ ) be $\Omega^{\prime}$ (resp. $\Omega$ ) and define a family of linear infinitesimally symplectic maps $B_{x}: R^{2 k} \rightarrow R^{2 k}$ (a local bundle change of coordinates) for $x \in V$ such that for all $u, v \in R^{2 k}$

$$
\Omega^{\prime}\left(u, B_{x} v\right)=\Omega_{x}(u, v) .
$$

Whenever $f$ is the local representative of a map in $\delta p_{\omega} \cap \delta p_{\omega^{\prime}}$ such that $f(x)=x$, we have for all $u, v \in R^{2 k}$

$$
\Omega^{\prime}\left(D f_{x} u, D f_{x} B_{x} v\right)=\Omega^{\prime}\left(D f_{x} u, B_{x} D f_{x} v\right)
$$

hence $D f_{x} B_{x}=B_{x} D f_{x}$.

Moreover, one shows easily that every linear symplectic map $A$ is given by the differential $D f_{x}$ of some $f \in \mathcal{S} p_{0}^{\prime}$ with $f(x)=x$. For example, if $x=0$ and $T$ is any symmetric matrix the time one map of the Hamiltonian flow generated by $H(x)=\frac{1}{2}\langle x, T x\rangle$ has differential $e^{J T}$ at zero $\left(J^{2}=-1\right)$. As $A=e^{J T}$ for appropriate $T, f$ is defined as the time one map for the Hamiltonian flow obtained by extending $H$ to all of $M$ with a suitable bump function. Thus, in view of the fact that $\delta p_{0}^{\prime} \subset \delta p_{0}, B_{x}$ commutes with the entire linear symplectic group of $\Omega^{\prime}$. This implies $B_{x}$ is a scalar matrix and that $\omega=F \omega^{\prime}$ for some nonvanishing function $F: M \rightarrow R$. But again, since $\delta p_{0}^{\prime} \subset \delta p_{0}, F \circ f=F$ for all $f \in \delta p_{0}^{\prime}$ and $F$ is a nonzero constant. We have thus shown that every $\omega^{\prime}$ is a constant multiple of $\omega$. By (iii), $\Sigma_{\omega}$ is a slice only if $H^{2}(M)=R$. In this case, conditions (i) and (ii) are obviously satisfied and condition (iii) follows from the remark after Theorem 2.2. Q.E.D.

REMARK. When $H^{2}(M)=R$, the slice $\Sigma_{\omega}$ is given by the open ray $\{t \omega\}_{t>0}$ in $Z^{2}$. Moreover, the map $\omega \rightarrow \omega^{k}$ is a $\mathscr{D}$-equivariant local submersion of the symplectic forms onto the volume elements which sends the slice $\Sigma_{\omega}$ to a slice $\Sigma_{\omega^{k}}$ for the group action on volumes.

EXAMPLE. A slice exists at each symplectic form on $\mathbf{C} P^{n}$ but not for $T^{2 n}$.

Since the proof of Theorem 2.3 depends on condition (ii) one might conjecture the existence of a "slice" which fails to satisfy this property. We can prove

Proposition 2.4. If the second Betti number of $M$ is $p>1$ there does not exist a manifold $\Sigma_{\omega}$ satisfying (i) $\delta p_{0} \cdot \Sigma_{\omega}=\Sigma_{\omega}$ and (ii) $\Sigma_{\omega}$ is transverse to the cohomology class $[\omega]$ of $\omega$ in $Z^{2}$.

Proof. Assume $\Sigma_{\omega}$ exists. Since $\Sigma_{\omega}$ is a manifold we can define $T_{\omega} \Sigma_{\omega}=E$ the tangent space to $\Sigma_{\omega}$ at $\omega$ and by (ii) $\operatorname{dim} E=p>1$. For $f \in \delta p_{0}, f^{*}$ acts linearly on 2-forms so the tangent map $T_{\omega}\left(f^{*}\right)=f^{*}$ and $f^{*}: E \rightarrow E$. As $f$ is homotopic to the identity $f^{*} \theta \in[\theta]$ for any closed 2-form, hence $\delta p_{0} \cdot \theta \subset$ $[\theta]$ for each $\theta \in E$. But, then $\delta p_{0} \cdot \theta \subset E \cap[\theta]$ and, since $E \cap[\theta]=\theta$ by the transversality, $\delta p_{0} \cdot \theta=\theta$. Now, for each element $\theta$ in a neighborhood of the origin in $E, \theta+\omega$ is symplectic. Set $\omega^{\prime}=\theta+\omega$ and note that for $f \in \delta p_{0}, f^{*} \omega^{\prime}=\omega^{\prime}$, i.e. $\delta p_{0} \subset \delta p_{\omega^{\prime}}$. By the argument given in Theorem 2.3, 
$\omega^{\prime}=t \omega$. This means $\alpha+\omega=t \omega$ or $\alpha=(t-1) \omega$ which implies $\operatorname{dim} E=1$. Q.E.D.

3. The global theory. In this section we enlarge the identity component $\mathscr{Q}_{0}^{s+1}$ to the full group of (orientation-preserving) diffeomorphisms $\mathscr{Q}^{s+1}$. The symbol $\Phi$ denotes, as before, the group of smooth diffeomorphisms of $M^{2 k}$.

Recall that in Theorem 2.2 we established the existence of a diffeomorphism

$$
\mathfrak{Q}_{0}^{s+1} / \delta p_{0}^{s+1} \cong \mathcal{O}_{0}^{s}=\left\{f^{*} \omega \mid f \in \mathscr{Q}_{0}^{s+1}\right\}
$$

for $s>k$ characterizing the orbit of the smooth form $\omega$ as a homogeneous space of the identity component of $\mathbb{Q}^{s+1}$. In this section one may work in the Sobolev $\left(\mathcal{H C}^{s}\right)$ or smooth $\left(\mathfrak{C}^{\infty}\right)$ category. In the latter case, the above diffeomorphism becomes a homeomorphism.

The global properties of the action $\omega \rightarrow f^{*} \omega$ are complicated by the presence of, possibly, an uncountable number of connected components in $\mathscr{D}$ (or $\mathscr{Q}^{s+1}$ ). Fortunately, as we will demonstrate, the global orbit $\theta_{\omega}^{s}=\left\{f^{*} \omega \mid f\right.$ $\left.\in \mathscr{Q}^{s+1}\right\}$ exhibits more uniformity.

To simplify our presentation, we introduce the subgroup of $\mathscr{Q}^{s+1}$,

$$
\mathcal{G}^{s+1}=\left\{f \in \mathscr{Q P}^{s+1} \mid\left[f^{*} \omega\right]_{s}=[\omega]_{s}\right\},
$$

Proposition 3.1. The coset space $\mathcal{G}^{s+1} / \delta p^{s+1}$ is homeomorphic to the open subset of $[\omega]_{s}$ given by $\vartheta_{g}^{s+1}=\mathcal{\theta}_{\omega}^{s+1} \cap[\omega]_{s}$, and consists of a union of open components homeomorphic to $\theta_{0}^{s}$.

Proof. We sketch the straightforward proof. Define the group

$$
\mathfrak{L}^{s+1}=\left\{f \in \mathscr{Q}^{s+1} \mid f^{*} \omega=g^{*} \omega, \text { for some } g \in \mathscr{T}_{0}^{s+1}\right\} .
$$

Then the coset map

$$
\mathscr{W}_{0}^{s+1} / \delta p_{0}^{s+1} \rightarrow \mathfrak{L}^{s+1} / \delta p_{\omega}^{s+1},
$$

is bijective, continuous, and open; i.e., a homeomorphism. But $\mathscr{D}_{0}^{s+1} / \delta p_{0}^{s+1}$ is homeomorphic to $\theta_{0}^{s+1}$ (Theorem 2.2) and the quotient $\mathcal{G}^{s+1} / \mathcal{S}_{p}^{s}$ is a union of open components homeomorphic to $\mathfrak{L}^{s+1} / \delta p_{\omega}^{s+1}$. Q.E.D.

The diffeomorphism group $\mathscr{P}^{s+1}$ induces an action on cohomology by defining for each $f \in \mathscr{Q}^{s+1}$ and $[\omega]_{s} \in H^{2}[M] ; f^{\#}[\omega]_{s}=\left[f^{*} \omega\right]_{s}$. Let $\mathcal{O}_{[\omega]}^{s}$ denote the orbit of this action-the set of cohomologically distinct equivalent symplectic structures.

THEOREM 3.2. The following assertions hold for the symplectic closed manifold M.

(1) $\Theta_{[\omega]}^{s}$ is a discrete submanifold of $H^{2}(M)$ homeomorphic to the quotient manifold $\mathscr{Q}^{s+1} / \mathcal{G}^{s+1}$.

(2) The global orbit $\mathcal{O}_{\omega}^{s}$ of forms equivalent to $\omega$ is naturally homeomorphic to the product $\theta_{[\omega]}^{s} \times \theta_{\Theta}^{s}$. 
Proof. (1) From the orbit principle for the group action $\left(\vartheta^{s+1}\right)^{\#}$ on $H^{2}(M)$, the sets $\mathscr{Q}^{s+1} / \mathcal{G}^{s+1}$ and $\theta_{[\omega]}^{s}$ are in one-to-one correspondence. We now show that $\Theta_{[\omega]}^{s}$ is a discrete (countable) submanifold, and so the bijection is a homeomorphism.

Since $\vartheta_{[\omega]}^{s}$ does not depend on " $s$ ", we delete it for simplicity. Let $p>1$ denote the second Betti number of $M$, and suppose $\Gamma=\left\{\Gamma_{i}\right\}_{i=1}^{p}$ is a basis for the maximal free submodule in $H_{2}(M, Z)$. Then $\Gamma$ is a basis for $H_{2}(M, R)$, and we may denote the corresponding dual basis for $\left(H_{2}(M, R)\right)^{*}$ as $\left\{\gamma_{i}\right\}_{i=1}^{p}$. By the de Rham Theorem, $[\omega]=\Sigma C_{i} \gamma_{i}$ where $C_{i}=\int_{\Gamma_{i}} \omega$. If $f$ is in $\mathscr{D}$, then [ $\left.f^{*} \omega\right]=\sum d_{i} \gamma_{i}$ with $d_{i}=\int_{\Gamma_{i}} f^{*} \omega=\int_{f \circ \Gamma_{i}} \omega$. As there exist integers $N_{k i}(f)$ such that

$$
f \circ \Gamma_{i}=\sum_{k} N_{k i}(f) \Gamma_{k}
$$

then

$$
d_{i}=\sum_{k} N_{k i} C_{k} \text { and }\left[f^{*} \omega\right]=\sum_{k i} N_{k i} C_{k} \gamma_{i} .
$$

Thus, assertion (1) is proved.

(2) This result is now immediate from Proposition 3.1 and the decomposition

$$
\mathcal{Q}^{s+1} / \delta p^{s+1} \cong \mathcal{Q}^{s+1} / \mathcal{G}^{s+1} \times \mathcal{G}^{s+1} / \delta p^{s+1} \text {. Q.E.D. }
$$

As a final remark, the reader might wish to contrast the symplectic forms with contact structures, which are stable under change of coordinates. Thus any one-form $\theta^{\prime}$ close to a one-form $\theta$ which induces a contact structure on $M=M^{2 k+1}$ is equivalent to a function multiple of $\theta$. This fact suggests a slice for such one-forms, and may provide a means of deciding whether a manifold admits a contact structure.

\section{REFERENCES}

1. D. Ebin, The manifold of Riemannian metrics, Proc. Sympos. Pure Math., vol. 15, Amer. Math. Soc., Providence, R. I., 1970, pp. 11-40.

2. D. Ebin and J. Marsden, Groups of diffeomorphisms and the motion of an incompressible fluid, Ann. of Math. (2) 92 (1970), $102-163$.

3. M. Golubitsky and D. Tischler, The nonexistence of globally stable forms, Proc. Amer. Math. Soc. 58 (1976), 296-300.

4. J. Martinet, Sur les singularités des formes différentielles, Ann. Inst. Fourier (Grenoble) 20 (1970), fasc. 1, 95-178.

5. D. Montgomery and L. Zippin, Topological transformation groups, Interscience, New York, 1955.

6. J. Moser, On the volume elements on a manifold, Trans. Amer. Math. Soc. 120 (1965), 286-294.

7. H. Omori, On the group of diffeomorphisms of a compact manifold, Proc. Sympos. Pure Math., vol. 15, Amer. Math. Soc., Providence, R. I., 1970, pp. 167-183.

8. R. Palais, On the existence of slices for actions of non-compact Lie groups, Ann. of Math. (2) 73 (1961), 295-323.

Department of Mathematics, Universtty of Missouri, Columbia, Missouri 65211 